\title{
A Performance Analysis of CLMS and Augmented CLMS Algorithms for Smart Antennas
}

\author{
Y. Rama Krishna ${ }^{1}$ P E S N Krishna Prasad ${ }^{2}$ P.V. Subbaiah ${ }^{3}$ \\ B. Prabhakara Rao ${ }^{4}$ \\ ${ }^{1 \& 2}$ Prasad V. Potluri Siddhartha Institute of Technology, Vijayawada, India \\ ramakrishna.yarlagadda@gmail.com \\ surya125@gmail.com \\ ${ }^{3}$ Amrita Sai Institute of Science \& Technology, Vijayawada, India \\ pvs_ece2000@yahoo.co.in \\ ${ }^{4}$ JNT University Kakinada, Kakinada, India \\ drbprerediffmail.com
}

\begin{abstract}
An adaptive beam former is a device, which is able to steer and modify an array's beam pattern in order to enhance the reception of a desired signal, while simultaneously suppressing interfering signals through complex weight selection. However, the weight selection is a critical task to get the low Side Lobe Level (SLL) and Low Beam Width. It needs to have a low SLL and low beam width to reduce the antenna's radiation/reception ability in unintended directions. The weights can be chosen to minimize the SLL and to place nulls at certain angles. A vast number of possible window functions that are available to provide the weights to be used in Smart Antennas. This paper presents various traditional windowing techniques such as Binomial, Kaiser-Bessel, Blackman, Gaussian, and so on for computing weights for adaptive beam forming and also neural based methods like, Least Mean Square (LMS), Complex LMS (CLMS) [5], and Augmented CLMS (ACLMS) [1] algorithms. This paper discusses about various observations on signal processing techniques of Smart Antennas, that compromise between SLL and beam width (Directivity), to improve the base station capacity in Cellular and Mobile Communications and also the performance analysis of CLMS and ACLMS in terms of SLL and beam width, error convergence rate.
\end{abstract}

\section{KEYWORDS}

Adaptive Array, Beamforming, Smart Antennas, Wireless Sensor Networks, Complex Least Mean Square (CLMS), Augmented CLMS (ACLMS), Side Lobe Level (SLL), Beam width, Error Convergence Rate.

\section{INTRODUCTION}

Wireless cellular networks $[9,10]$ are fast growing technology in the current world and this trend is likely to continue for several years. The advancements in radio technology enable novel and improved services in the cellular systems. Current wireless services include transmission of voice, fax and Multimedia applications and so on. Multimedia services like video-on demand and internet access needs more band width. Wireless networks must provide these services in a wide range of environments, spanning dense urban, suburban and rural areas. Mobility needs varying must also be addressed.

Sundarapandian et al. (Eds): CoNeCo,WiMo, NLP, CRYPSIS, ICAIT, ICDIP, ITCSE, CS \& IT 07, pp. 09-19, 2012. (C) CS \& IT-CSCP 2012

DOI : $10.5121 /$ csit.2012.2402 
Smart Antennas (SA) $[11,15]$ consists of an array of antenna elements with signal processing capability that optimizes the radiation and reception of a desired signal dynamically. SAs can place nulls in the direction of interferers via adaptive updating of weights linked to each antenna element. SAs [14], thus cancel out most of the co-channel interference resulting the better quality of reception and lowered dropped calls. SAs can also track the user within a cell via direction of arrival algorithms.

The Smart antennas $[11,13]$ perform spatial filtering, which makes it possible to receive energy from a particular direction, while simultaneously blocking it from another direction. This property makes smart antennas as an effective tool in detecting and locating radiation from other sources. That means, the design and development of the efficient models to this task for real time optimization is a current problem. The control unit of the Smart Antenna is normally realized with a Digital Signal Processing (DSP) unit. The DSP controls radiation parameters of the antenna, based on certain inputs, in order to optimize the communication link. Figure 1 shows the basic model of Smart Antenna System.

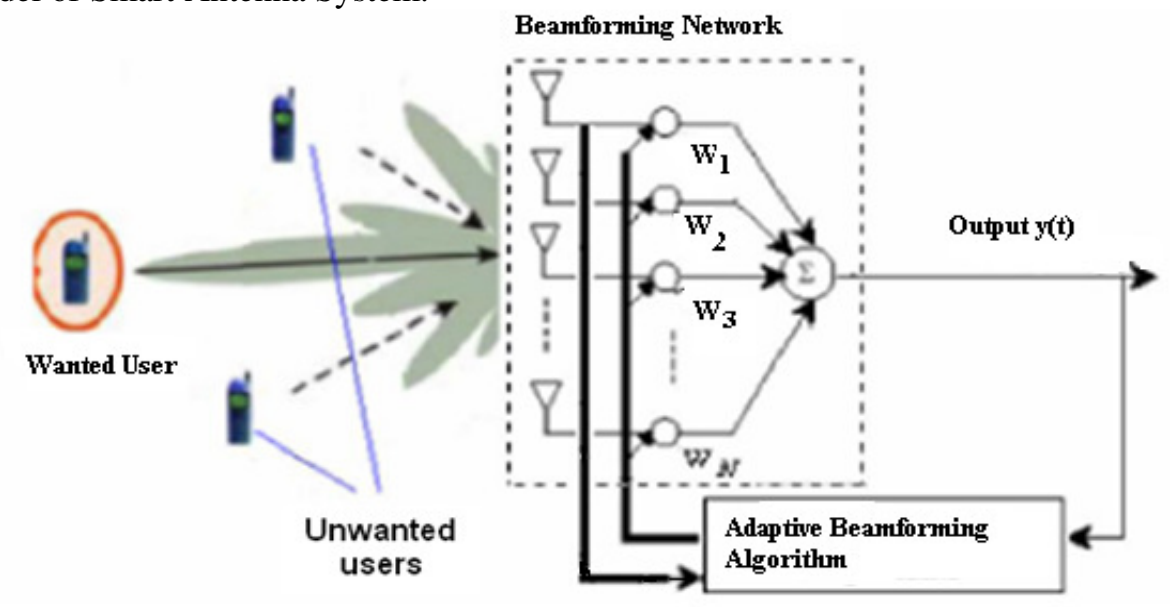

Figure 1. Basic smart antenna system

\section{COMPleX Neural AlgorithmS}

A Complex valued Neural Network $[2,7,12]$ is an artificial neural network, consists of complex valued input signals, weights, threshold values and/or signal functions. Such kind of models must be needed for solving the problems in the field of signal processing. In the signal processing, signals are complex valued and processing of such signals requires the implementation of new complex valued neural processing models. One of the most important characteristics of the complex valued neural models process linear complex signals of the smart antennas. In smart antennas signals from different sources or interferers are to be processed before orienting the main beam direction of the antenna array. In this context identifying the angle of arrival of the desired signal is very important. More over the Half Power Beam Width (HPBW) of the array radiation pattern must be as small as possible to avoid the interference. Similarly the Side Lobe Level (SLL).

In this paper Complex Least Mean Square (CLMS) and Augmented Complex Least Mean Square (ACLMS) algorithms $[1,5]$ have been considered as complex valued neural networks that can be applied on complex signals of Smart Antenna System.

Least Mean Square (LMS) is fundamental gradient based algorithm introduced by Widrow and Hoff in 1959 that estimates the gradient vector from the available data. This algorithm is an iterative method that leads to Minimum Mean Square Error (MSE), but this is a simple model which cannot process complex data with more noise. In the analysis of LMS, it is observed that, 
its convergence is slow if the eigen values are widely spread and it is directly depends on the eigen structure. The convergence time of LMS can be exceedingly long and highly data dependent when the eigen values of the covariance matrix differs.

In order to process such complex signals, variants of LMS model such as Complex Least Mean Square (CLMS) and Augmented Complex Least Mean Square (ACLMS) algorithms were chosen and the performance analysis of these models on complex signals is presented in section 3.

\subsection{Complex Least Mean Square Algorithm (CLMS)}

Complex Least Mean Square [5, 6, 8] algorithm was introduced by Widrow et al. in 1975, which benefits from the robustness and stability of the LMS and enables the simultaneous processing of complex signals.

This algorithm performs stochastic gradient decent in complex domain statistics that enables better modeling of complex data and produce effective outcome. The basic algorithm of CLMS is as follows:

1. The instantaneous estimates

$$
\begin{aligned}
& E\left[\left|e^{2}(k)\right|\right] \rightarrow \frac{1}{2}\left|e^{2}(k)\right| \\
& E\left[x(k) x^{H}(k)\right] \rightarrow x(k) x^{H}(k) \\
& E[x(k) d(k)] \rightarrow x(k) d(k)
\end{aligned}
$$

2. The 'stochastic' cost function

$$
J(k)=\frac{1}{2}|e(k)|^{2}
$$

3. Weight vector update

$$
w(k+1)=w(k)-\mu \nabla_{w} J(w)
$$

4. The gradient of the cost function

The gradient of the cost function with respect to the complex valued weight vector $w(k)=w_{r}(k)+j w_{i}(k)$ can be expressed as

$$
\nabla_{\mathrm{w}} \mathrm{J}(\mathrm{k})=\nabla_{\mathrm{w}_{\mathrm{r}}} \mathrm{J}(\mathrm{k})+\mathrm{j} \nabla_{\mathrm{w}_{\mathrm{i}}} \mathrm{J}(\mathrm{k})=\frac{\partial \mathrm{J}(\mathrm{k})}{\partial \mathrm{w}_{\mathrm{r}}(\mathrm{k})}+\mathrm{j} \frac{\partial \mathrm{J}(\mathrm{k})}{\partial \mathrm{w}_{\mathrm{i}}(\mathrm{k})}
$$

5. The output error is given by

$$
e(k)=d(k)-x^{T}(k) w(k)
$$

6. The stochastic gradient adaptation for the weight vector can be expressed as

$$
\mathrm{w}(\mathrm{k}+1)=\mathrm{w}(\mathrm{k})+\mu \mathrm{e}(\mathrm{k}) \mathrm{x}^{*}(\mathrm{k}), \mathrm{w}(0)=0
$$

7. This output of the complex least mean square (CLMS) algorithm is computed as

$$
y=x^{H}(k) w(k) \rightarrow w(k+1)=w(k)+\mu e(k) x(k)
$$

\subsection{Augmented Complex Least Mean Square Algorithm (ACLMS)}

The ACLMS $[1,5,8]$ algorithm has the same generic form as the standard CLMS, it is simple to implement, yet it takes into account the full available second-order statistics of complex valued inputs (non circularity) in the domain of adaptive beam forming that utilizes the second order statistical information. This is achieved based on some advancement in complex statistics with the use of widely linear modeling. So this model is also called as widely Linear LMS. The ACLMS has advantages over CLMS including: 
1. In blind source separation, it may be able to deal with more sources than observations.

2. Improved signal recovery in communications modulation schemes.

3. Improved direction of arrival estimation in augmented array signal processing.

4. The analysis of augmented signal processing algorithms benefits from special matrix structures which do not exist in standard complex valued signal processing.

The basic algorithm of ACLMS is as follows:

1. The output of ACLMS is

$$
\mathrm{y}(\mathrm{k})=\sum_{\mathrm{n}=1}^{\mathrm{N}}\left[\mathrm{h}_{\mathrm{n}}(\mathrm{k}) \mathrm{z}(\mathrm{k}-\mathrm{n})+\mathrm{g}_{\mathrm{n}}(\mathrm{k}) \mathrm{z}^{*}(\mathrm{k}-\mathrm{n})\right] \Leftrightarrow \mathrm{y}(\mathrm{k})=\mathrm{h}^{\mathrm{T}}(\mathrm{k}) \mathrm{z}(\mathrm{k})+\mathrm{g}^{\mathrm{T}}(\mathrm{k}) \mathrm{z}^{*}(\mathrm{k})
$$

2. Weight updation:

$$
\Delta \mathrm{w}_{\mathrm{n}}(\mathrm{k})=-\mu \nabla \mathrm{w}_{\mathrm{n}} \mathrm{J}(\mathrm{k})=-\mu \frac{\partial J(\mathrm{k})}{\partial \mathrm{w}_{\mathrm{n}}(\mathrm{k})}=-\mu\left(\frac{\partial \mathrm{J}(\mathrm{k})}{\partial \mathrm{w}_{\mathrm{n}}^{\mathrm{r}}(\mathrm{k})}+\mathrm{j} \frac{\partial \mathrm{J}(\mathrm{k})}{\partial \mathrm{w}_{\mathrm{n}}^{\mathrm{i}}(\mathrm{k})}\right)
$$

Where $w_{n}(k)=w_{n}^{r}(k)+j w_{n}^{i}(k)$, is a complex weight and $\mu$ is the learning rate, a small positive constant.

$$
\begin{aligned}
& h(k+1)=h(k)+\mu e(k) z^{*}(k) \\
& g(k+1)=g(k)+\mu e(k) z(k)
\end{aligned}
$$

3. The augmented weight vector

$$
w^{a}(k)=\left[h^{T}(k), g^{T}(k)\right]^{T}
$$

4. The final form of ACLMS as follows:

$$
w^{a}(k+1)=w^{a}(k)+\mu e(k) z^{a *}(k)
$$

where the 'augmented' instantaneous error is

$$
e(k)=d(k)-z^{a T}(k) w^{a}(k)
$$

\section{Performance Analysis}

In the process of adaptive beam forming signals [14, 15], the input signal is considered as $x_{s}(k)=\cos (2 w t)$ with frequency $1 \mathrm{kHz}$ and a noise is also considered along with the input signal. Normalized weights are calculated using different algorithms such as Binomial, Blackman, Hamming, Gaussian and Kaiser-Bessel. The Half Power Beam width and SLL are calculated from the array factors plotted with the normalized weights of the above algorithms and presented in the table 1. From the tabular values, it is observed that there is a compromise between HPBW and SLL.

Table 1. HPBW and SLL value comparison for traditional algorithms.

\begin{tabular}{|c|c|c|c|c|}
\hline S.No. & Weights Used & HPBW (Degrees) & $\begin{array}{c}\text { No of Symmetrical } \\
\text { Side Lobes }\end{array}$ & SLL \\
\hline 1 & Un Weighted & 12.82 & 3 & 0.2291 \\
\hline 2 & Binomial & 22.95 & 0 & 0 \\
\hline 3 & Blackman & 27.21 & 0 & 0 \\
\hline 4 & Hamming & 20.43 & 1 & 0.0210 \\
\hline 5 & Gaussian & 19.73 & 1 & 0.0084 \\
\hline 6 & Kaiser-Bessel & 16.98 & 3 & 0.0372 \\
\hline
\end{tabular}

Later, the complex valued neural models CLMS and ACLMS algorithms are chosen for adaptive beam forming algorithms to calculate the weights for the same input signals with different values 
for the parameters such as number of array elements $(\mathrm{N})$ and Step Size $(\mu)$. Among the list the better results have been considered without noise and presented in table 2.

Table 2. Performance analysis of CLMS \& ACLMS algorithms without noise.

\begin{tabular}{|c|c|c|c|c|c|c|}
\hline SL. No & $\begin{array}{c}\text { Weights } \\
\text { Used }\end{array}$ & $\begin{array}{c}\text { No. of Array } \\
\text { Elements }(\mathrm{N})\end{array}$ & $\begin{array}{c}\text { Step size } \\
\text { Parameter }(\mu)\end{array}$ & $\begin{array}{c}\text { HPBW } \\
\text { (Degrees) }\end{array}$ & $\begin{array}{c}\text { No of } \\
\text { symmetrical } \\
\text { Side Lobes }\end{array}$ & SLL \\
\hline 1 & CLMS & 8 & 0.015 & 7.90 & 6 & 0.0654 \\
\hline 2 & CLMS & 8 & 0.025 & 9.20 & 3 & 0.0453 \\
\hline 3 & CLMS & 8 & 0.050 & 13.20 & 3 & 0.0799 \\
\hline 4 & ACLMS & 8 & 0.015 & 9.90 & 2 & 0.0429 \\
\hline 5 & ACLMS & 8 & 0.025 & 13.40 & 5 & 0.0799 \\
\hline 6 & ACLMS & 8 & 0.050 & 20.0 & 6 & 0.1408 \\
\hline
\end{tabular}

From table 2 it can be observed that, when CLMS and ACLMS weights are used, there is a considerable reduction of HPBW and SLL. Corresponding data is also presented in figures 2, 3 and 4. From the observation of figure 2 with the use of CLMS, minimum value for HPBW and least SLL at $\mathrm{N}=9$ and $\mu=0.015$ has been obtained. Similarly from the figure 3 with the use of ACLMS, minimum value for HPBW and least SLL at N=8 and $\mu=0.015$ has been obtained. From these two observations CLMS has given better values for HPBW and SLL which is presented in figure 4. The convergence of array outputs towards the desired signal computed using CLMS and ACLMS is also presented in figure 5. From figure 5, it is observed that ACLMS gives nearest convergence towards the desired signal than CLMS.

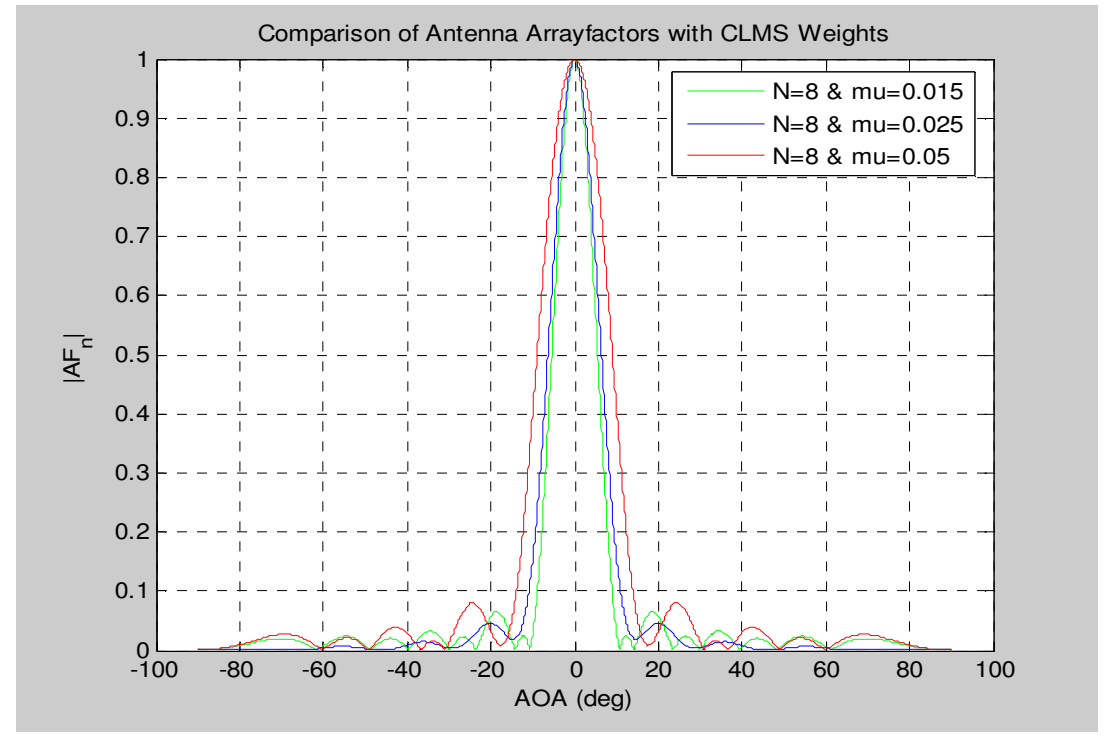

Figure 2. Comparison of performance of CLMS weights in noiseless environment

In order to simulate the real time environment of Smart Antenna System, the noise component has been considered in addition to the input signal and the performance of the CLMS and ACLMS algorithms have been analysed with different values of $N$ and $\mu$. The results obtained with noise are presented in table 3. However it is observed that, the reduction of step size parameter $\mu$ improved the performance of the chosen algorithm in noisy environment rather than noiseless environment. The analysis of these two algorithms is presented in figure 6 (CLMS, 
$\mathrm{N}=8$ ), 7 (CLMS, N=9), 8 (ACLMS, N=8), 9 (ACLMS, N=9) and 10(Best Performance of CLMS and ACLMS with Noise). From this analysis, ACLMS gives best performance rather CLMS in the Noise, but the HPBW is almost similar. So, it is concluded that ACLMS gives better outcome in the noisy environment, where as in noiseless environment CLMS is better one.

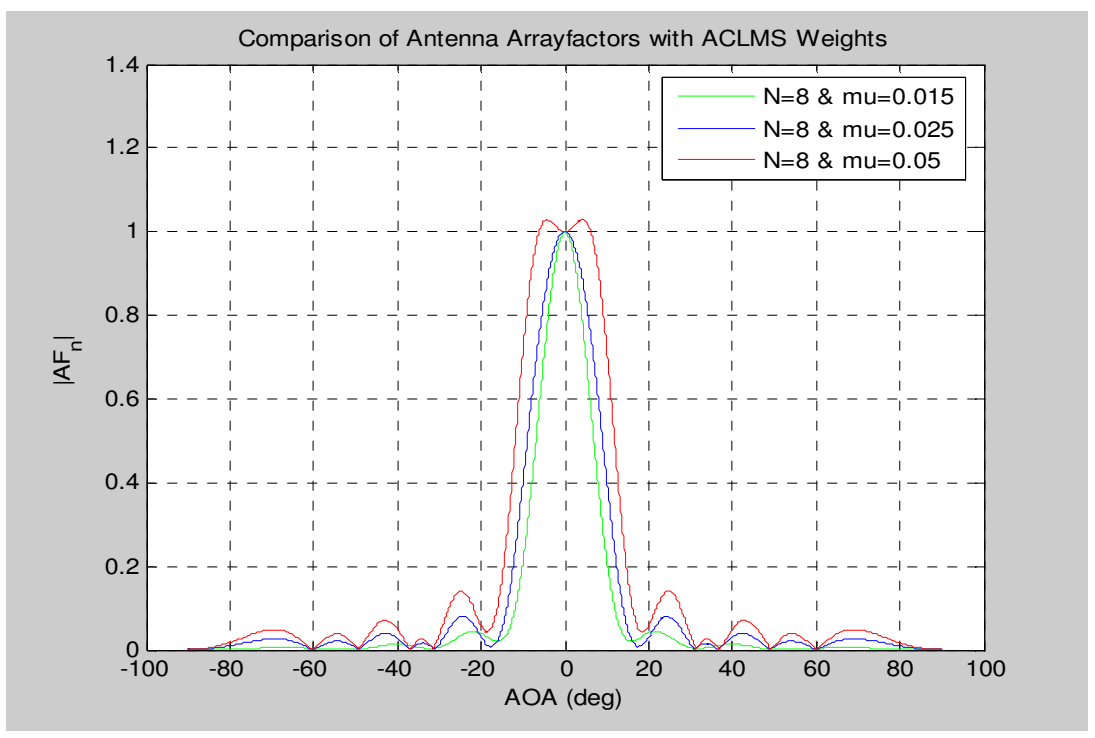

Figure 3. Comparison of performance of ACLMS weights in noiseless environment

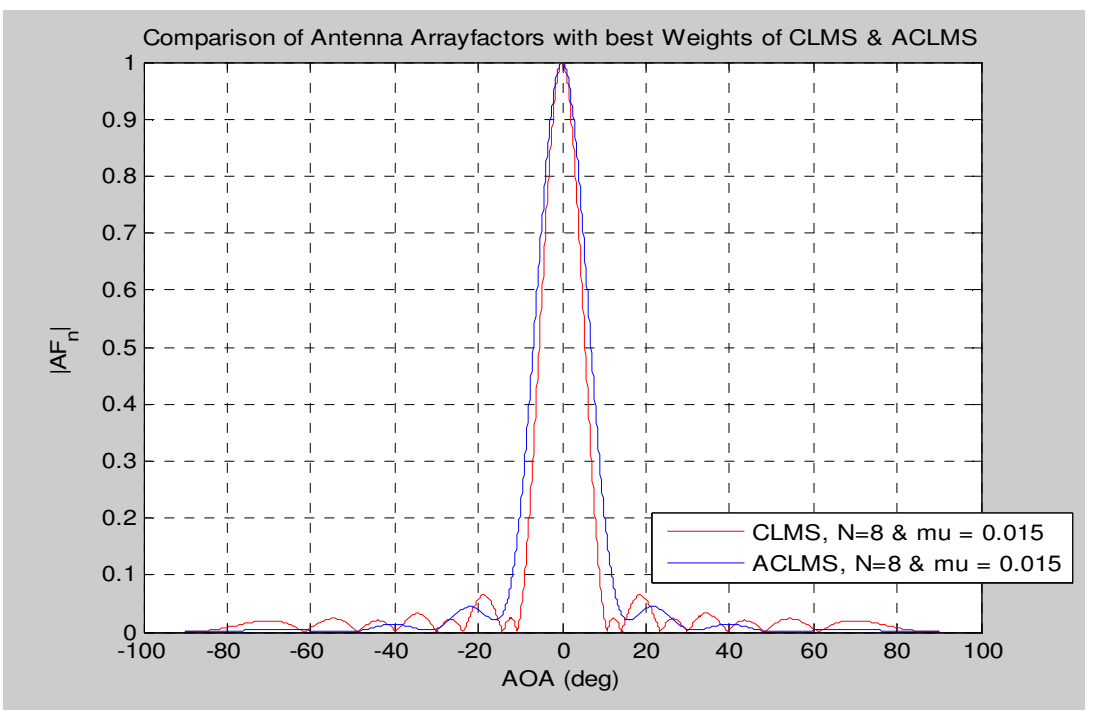

Figure 4. Comparison of best performance by CLMS \& ACLMS weights in noiseless environment 


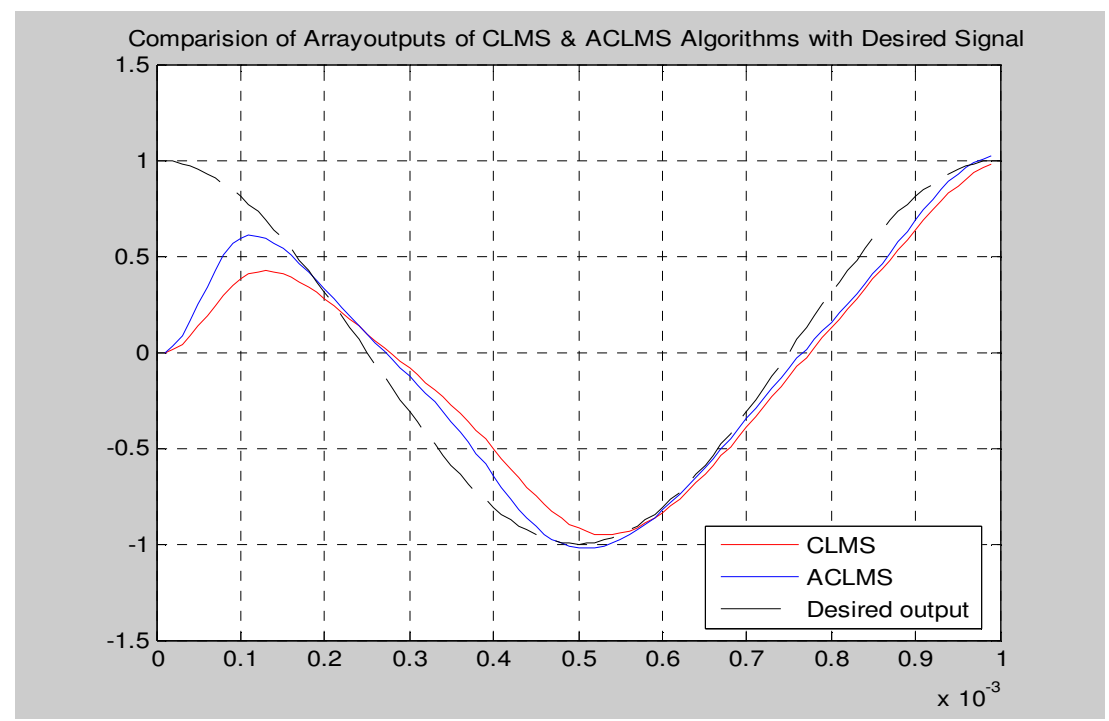

Figure 5. Signal convergence comparison

Table 3. Performance Analysis of CLMS \& ACLMS Algorithms with Noise

\begin{tabular}{|c|c|c|c|c|c|c|}
\hline Sl. No. & $\begin{array}{c}\text { Weights } \\
\text { Used }\end{array}$ & $\begin{array}{c}\text { No. of Array } \\
\text { Elements (N) }\end{array}$ & $\begin{array}{c}\text { Step size } \\
\text { Parameter }(\boldsymbol{\mu})\end{array}$ & $\begin{array}{c}\text { HPBW } \\
\text { (Degrees) }\end{array}$ & $\begin{array}{c}\text { No of } \\
\text { symmetrical } \\
\text { Side Lobes }\end{array}$ & SLL \\
\hline 1 & CLMS & 8 & 0.002 & 6.6 & 7 & 0.1555 \\
\hline 2 & CLMS & 9 & 0.002 & 6.1 & 6 & 0.1781 \\
\hline 3 & ACLMS & 8 & 0.002 & 6.4 & 6 & 0.1873 \\
\hline 4 & ACLMS & 9 & 0.002 & 6.5 & 6 & 0.1189 \\
\hline
\end{tabular}

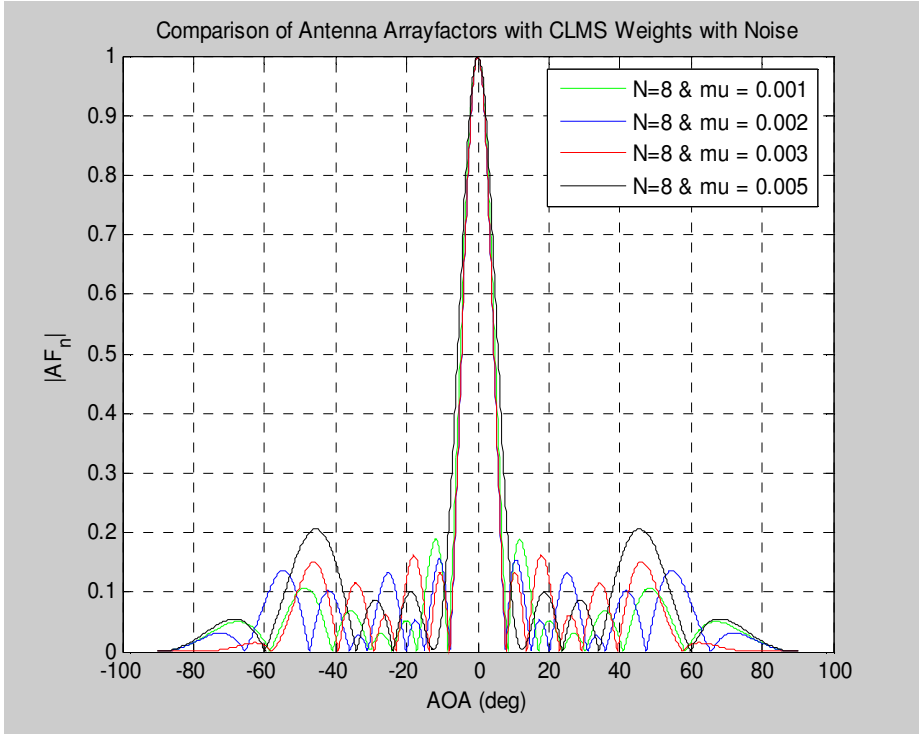

Figure 6. Comparison of performance of CLMS weights with $\mathrm{N}=8$ in noisy environment 


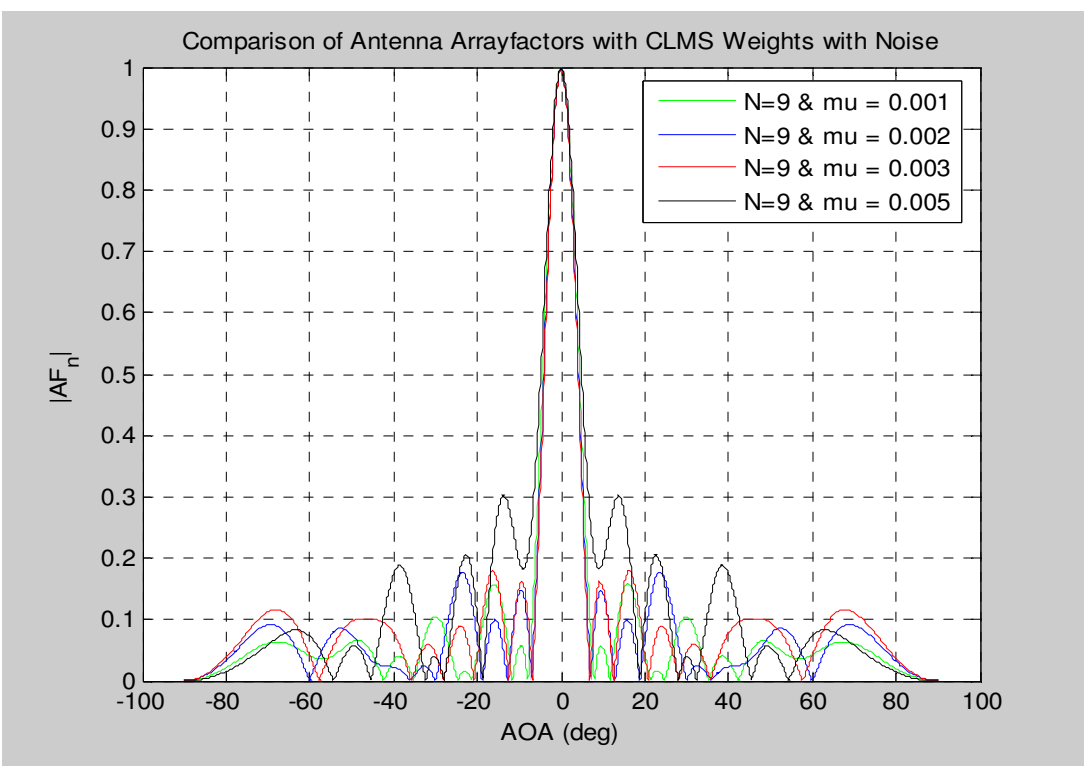

Figure 7. Comparison of performance of CLMS weights with $\mathrm{N}=9$ in noisy environment

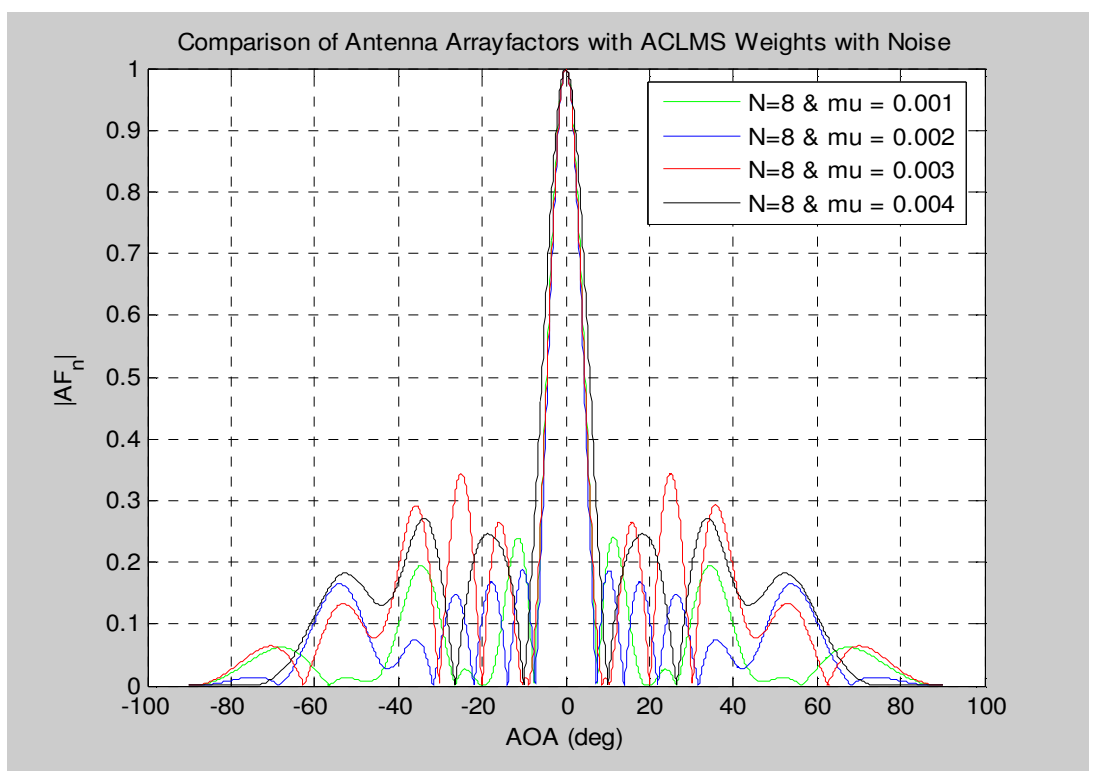

Figure 8. Comparison of performance of ACLMS Weights with $\mathrm{N}=8$ in noisy environment 


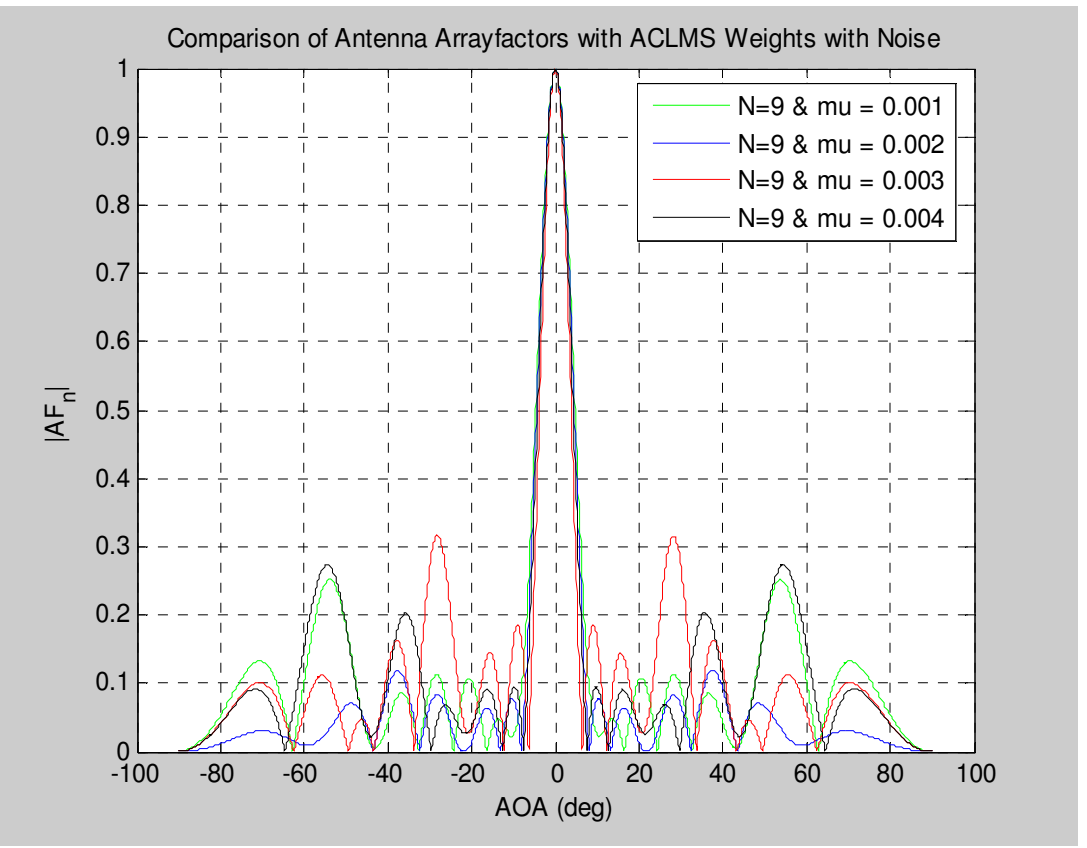

Figure 9. Comparison of performance of ACLMS Weights with $\mathrm{N}=9$ in noisy environment

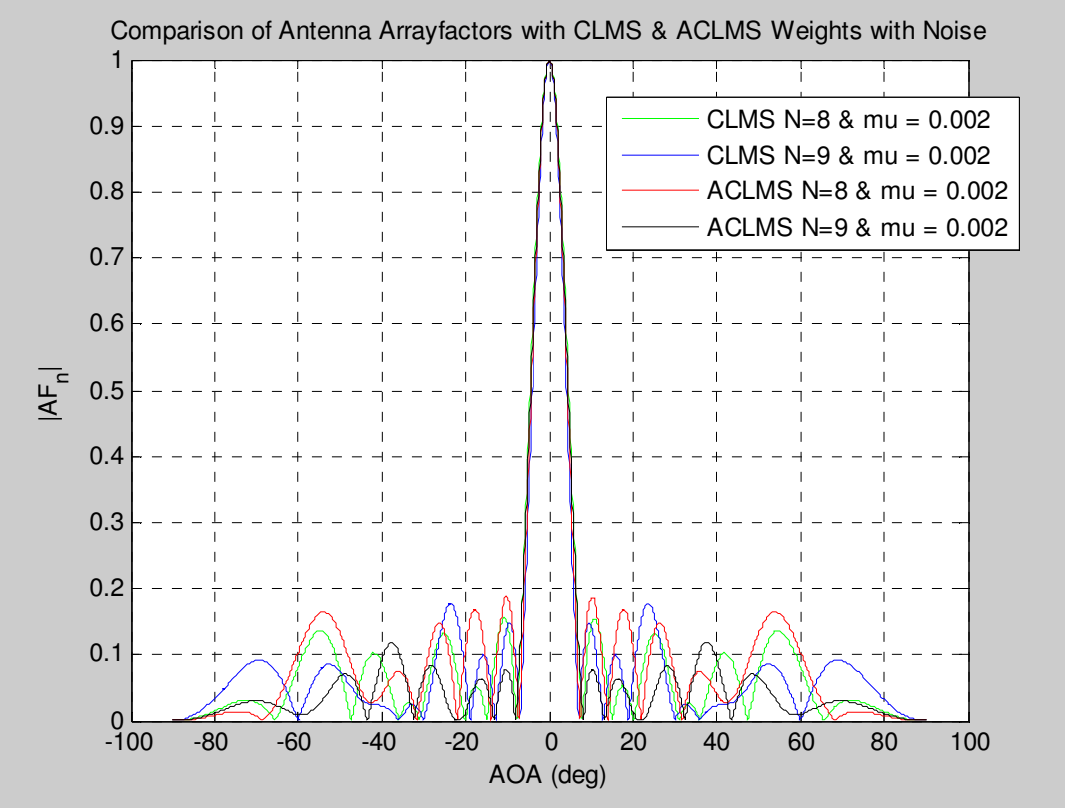

Figure 10. Comparison of best performance by CLMS \& ACLMS weights in noisy environment

\section{CONCLUSION}

As per considering the complex valued neural networks like CLMS and ACLMS on adaptive beamforming signals in Smart Antennas, various parameters such as number of array elements $(\mathrm{N})$, learning rate $(\mu)$ have been considered under noiseless and noisy environments. Based on such data, ACLMS is a better model than CLMS in the convergence towards desired signal, but 
CLMS is better model than ACLMS in giving low HPBW and SLL in noiseless environment, whereas in case of noisy environment ACLMS gives good performance in respect of HPBW and SLL but SLL has been increased. In order to improve the overall performance of Smart Antenna System by making use of the individual best aspect of CLMS and ACLMS algorithms, a new approach can be proposed by combining these two models as hybrid to minimize the SLL in noisy environment.

\section{REFERENCES}

[1] Soroush Javidi, Maciej Pedzisz, Su Lee Goh and Danilo P. Mandic, "The Augmented Complex Least Mean Square Algorithm With Application to Adaptive Prediction Problems", Proc. 1st IARP Workshop on Cognitive Information Processing, 2008, 54-57.

[2] D. Mandic, P. Vayanos, C. Boukis, B. Jelfs, S.L. Goh, T. Gautama, and T. Rutkowski, "Collaborative Adaptive Learning Using Hybrid Filters", ICASSP 2007, 2007, vol. 3, pp. 921-924.

[3] P. J. Schreier and L. L. Scharf, "Second-Order Analysis of Improper Complex Random Vectors and Processes", IEEE Transactions on Signal Processing, Vol. 51, No, 3. pp. 714-725, 2003.

[4] D. P. Mandic, S. Javidi, G. Souretis and S. L. Goh, "Why a Complex Valued Solution for a Real Domain Problem", Proceedings of the $17^{\text {th }}$ IEEE signal Processing Society Workshop on Machine Learning for Signal Processing, 2007.

[5] D. P. Mandic, Yili Xia and S. C. Douglas, "Steady State Analysis of the CLMS and Augmented CLMS Algorithms for Non-Circular Complex Signals", Proceedings of ASILOMAR, 2010, pp. 1635-1639.

[6] D. P. Mandic, Yili Xia and Ali H Syad, "An Adaptive Diffusion Augmented CLMS algorithm for Distributed Filtering of Non-Circular Complex Signals”, IEEE Signal Processing Letters, Vol. 18, No. $11,2011$.

[7] S. Haykin and Liang Li, "Nonlinear Adaptive Prediction of Non-Stationary Signals" IEEE Transactions on Signal Processing, Vol. 43, No. 2, pp. 526-535, 1995.

[8] D. P. Mandic and Vanessa Su Lee Goh, "Complex Valued Nonlinear Adaptive Filters - Noncircularity, Widely Linear and Neural Models", John Wiley \& Sons Ltd., 2009.

[9] Jack H. Winters, "Smart Antennas for Wireless Systems", IEEE Personal Communications, Vol. 5, No. 1, pp. 23-27, 1998.

[10] L.C. Godara, "Applications of Antenna Arrays to Mobile Communications. I. Performance Improvement, Feasibility and Considerations", IEEE Proceedings, Vol. 85, No.7, pp. 1031-1060, 1997.

[11] Ivica Stevanovic, Anja Skrivervik and Juan R. Mosig, "Smart Antenna Systems for Mobile Communications", Laboratoire d'Electromagnetisme et d'Acoustique Ecole Polytechnique Federale de Lausanne, 2003.

[12] Akira Hirose, "Complex valued Neural Networks: Theories and Applications", World Scientific Publications, 2003.

[13] Simon Haykin and Thomas Kailath, "Adaptive Filter Theory", Fourth Edition, Pearson Education, 2009.

[14] Smart Antennas - Beamforming Tutorial", www.altera.com

[15] "Smart Antenna Systems Tutorial", The International Engineering Consortium, www.iec.org 
Authors

Y. Ramakrishna currently pursuing Ph.D. Degree from JNTU Kakinada in the field of Smart Antennas for Mobile Communications. He received M.Tech Degree in Microwave Engineering from Acharya Nagarjuna University, India in 2005. Currently He is working as Associate Professor in the Department of ECE, Prasad V. Potluri Siddhartha Institute of Technology, India. He is a Member of ISTE. His Research interest includes Smart Antennas, Antennas and Wave Propagation, Mobile Communications and Microwave Engineering.

P. E. S. N. Krishna Prasad currently a Researcher in the area of Machine Intelligence and Neural Networks. He is working in Computer Applications at Prasad V. Potluri Siddhartha Institute of Technology, Vijayawada, Andhrapradesh, India. $\mathrm{He}$ is a member of ACM, IEEE, ISTE. He has presented and published papers in several International Conferences and Journals. His areas of interest include Artificial Intelligence, Neural Networks and Machine Intelligence, Computational Intelligence and Information security Applications.

Dr. P. V. Subbaiah received his Ph.D. in Microwave Antennas from JNTU, India, 1995. His Master's Degree in Control Systems from Andhra University, India, 1982. $\mathrm{He}$ is Currently Working as Principal at Amrita Sai Institute of Science and Technology, Vijayawada, India since 2007. His Research interest includes Microwave Antennas, Optical Communications and Mobile Communications.

Dr.B.Prabhakara Rao has more than 28 years of experience in teaching and 20 years of $\mathrm{R} \& \mathrm{D}$. He is an expert in Signal Processing \& Communications. He produced 5 $\mathrm{Ph}$. D's and guiding $25 \mathrm{Ph}$. D scholars. He held different positions in his career like Head of the Department, Vice Principal, in JNTU College of Engineering and Director (Institute of Science \& Technology) in the Newly Established JNT University from 2003 to 2010. Currently he is working as Director (Foreign Universities) at JNTU Kakinada. He published more than 95 technical papers in national and International journals and conferences.
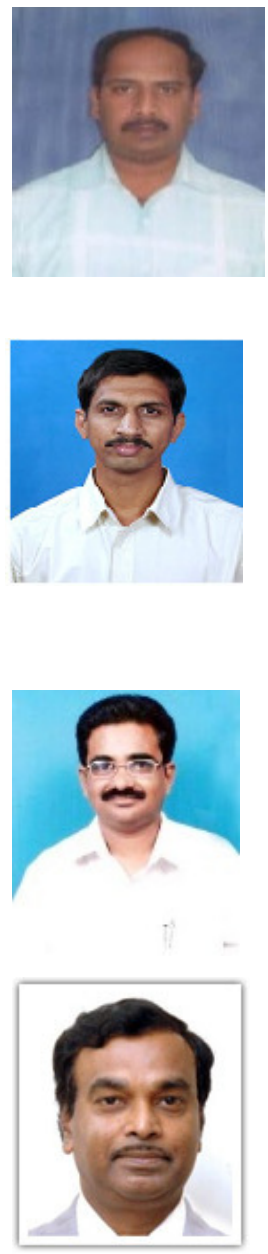